University of Wollongong

Research Online

Australian Institute for Innovative Materials -

Papers

Australian Institute for Innovative Materials

$1-1-2020$

\title{
Non-zero spontaneous magnetic moment along crystalline $b$-axis for rare earth orthoferrites
}

\author{
Mohanad Hazim Mohammed \\ University of Wollongong, mhm670@uowmail.edu.au \\ Zhenxiang Cheng \\ University of Wollongong, cheng@uow.edu.au \\ Shixun Cao \\ Kirrily C. Rule \\ University of Wollongong \\ Christopher Richardson \\ University of Wollongong, crichard@uow.edu.au
}

See next page for additional authors

Follow this and additional works at: https://ro.uow.edu.au/aiimpapers

Part of the Engineering Commons, and the Physical Sciences and Mathematics Commons

Research Online is the open access institutional repository for the University of Wollongong. For further information contact the UOW Library: research-pubs@uow.edu.au 


\title{
Non-zero spontaneous magnetic moment along crystalline b-axis for rare earth orthoferrites
}

\author{
Abstract \\ (C) 2020 Author(s). Rare earth orthoferrites demonstrate great application potentials in spintronics and \\ optical devices due to their multiferroic and magnetooptical properties. In RFeO3, magnetic R3+ undergo \\ a spontaneous spin reorientation in a temperature range determined by $\mathrm{R}$ (rare earth), where the magnetic \\ structure changes from $\Gamma 2$ to $\Gamma 4$. The b-axis component of their magnetic moment, $\mathrm{Mb}$, is reported in \\ numerous neutron diffraction studies to remain zero at all temperatures. More sensitive magnetometer \\ measurements reveal a small non-zero $\mathrm{Mb}$, which is minute above $\sim 200 \mathrm{~K}$. Mb increases with cooling and \\ reaches values of $\sim 10-3 \mu \mathrm{B} / \mathrm{f}$.u. at temperatures within or below the spin reorientation temperatures. Our \\ results can be explained by assuming the $\mathrm{Fe} 3+$ spins as the origin of non-zero $\mathrm{Mb}$, while $\mathrm{R} 3+$ spins \\ suppress $\mathrm{Mb}$. The representation analysis of point groups shows that non-zero $\mathrm{Mb}$ is associated with a \\ small admixture of the $\Gamma 3$ phase to $\Gamma 2$ or $\Gamma 4$. Such a mixing of the three magnetic phases requires at least \\ a fourth order of the spin Hamiltonian for $\mathrm{RFeO} 3$ to describe the non-zero $\mathrm{Mb}$

\section{Disciplines} \\ Engineering | Physical Sciences and Mathematics

\section{Publication Details} \\ Mohammed, M. H., Cheng, Z. X., Cao, S., Rule, K. C., Richardson, C., Edwards, A., Studer, A. J. \& Horvat, J. \\ (2020). Non-zero spontaneous magnetic moment along crystalline b-axis for rare earth orthoferrites. \\ Journal of Applied Physics, 127 (11), 113906-1-113906-12.

\section{Authors} \\ Mohanad Hazim Mohammed, Zhenxiang Cheng, Shixun Cao, Kirrily C. Rule, Christopher Richardson, A \\ Edwards, Andrew J. Studer, and Josip Horvat
}




\section{Non-zero spontaneous magnetic moment along crystalline $b$-axis for rare earth orthoferrites}

Cite as: J. Appl. Phys. 127, 113906 (2020); https://doi.org/10.1063/1.5115518

Submitted: 19 June 2019 . Accepted: 05 February 2020 . Published Online: 20 March 2020

Mohanad H. Mohammed (D), Z. X. Cheng (D), Shixun Cao (D, K. C. Rule (D), C. Richardson (D), A. Edwards, A. J. Studer, and J. Horvat (D)
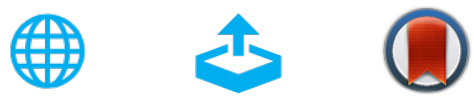

View Online

Export Citation

\section{ARTICLES YOU MAY BE INTERESTED IN}

Epitaxial growth and orientation-dependent anomalous Hall effect of noncollinear antiferromagnetic $\mathrm{Mn}_{3} \mathrm{Ni}_{0.35} \mathrm{Cu}_{0.65} \mathrm{~N}$ films

Journal of Applied Physics 127, 113907 (2020); https://doi.org/10.1063/1.5142250

Analysis of the magnetic and magnetocaloric properties of $\mathrm{ALaFeMnO}_{6}(\mathrm{~A}=\mathrm{Sr}, \mathrm{Ba}$, and $\mathrm{Ca})$ double perovskites

Journal of Applied Physics 127, 113905 (2020); https://doi.org/10.1063/1.5144153

High temperature piezoelectric response of polycrystalline Li-doped $(\mathrm{K}, \mathrm{Na}) \mathrm{NbO}_{3}$ ceramics under compressive stress

Journal of Applied Physics 127, 114101 (2020); https://doi.org/10.1063/1.5134554

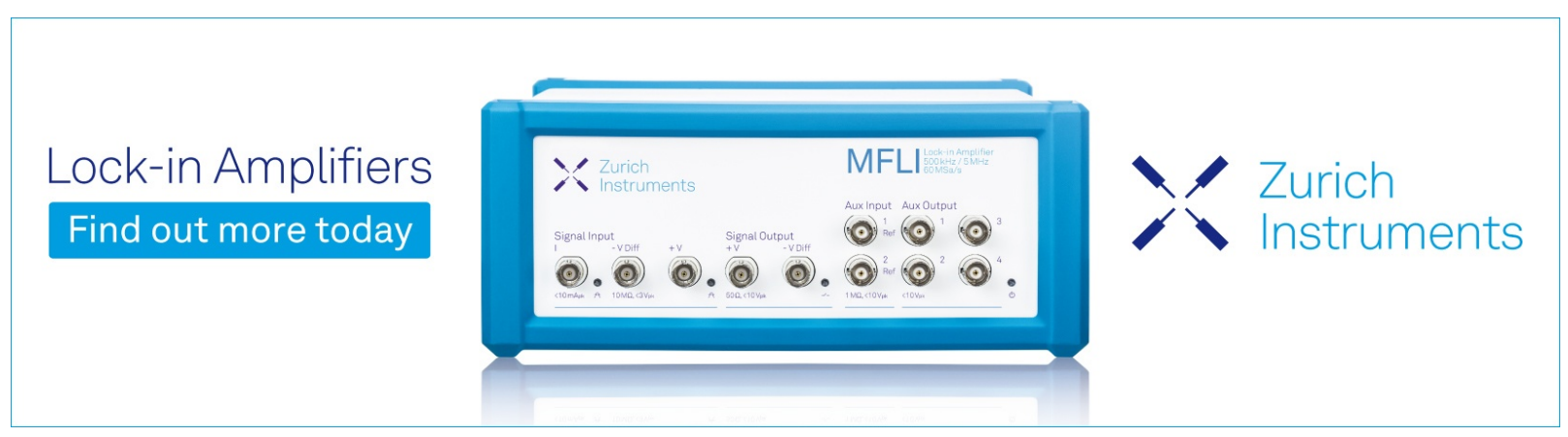




\title{
Non-zero spontaneous magnetic moment along crystalline $b$-axis for rare earth orthoferrites
}

\author{
Cite as: J. Appl. Phys. 127, 113906 (2020); doi: 10.1063/1.5115518 \\ Submitted: 19 June 2019 . Accepted: 5 February 2020 . \\ Published Online: 20 March 2020
}

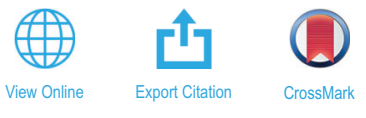

Mohanad H. Mohammed, ${ }^{1}$ (D) Z. X. Cheng, ${ }^{1, a)}$ (D) Shixun Cao, ${ }^{2}$ (D) K. C. Rule, ${ }^{1,3}$ (D) C. Richardson, ${ }^{4}$ (D) A. Edwards, ${ }^{3}$ A. J. Studer, ${ }^{3}$ and J. Horvat ${ }^{1, b)}$ (D)

\author{
AFFILIATIONS \\ ${ }^{1}$ Institute for Superconducting and Electronic Materials and School of Physics, University of Wollongong, Wollongong, \\ NSW 2522, Australia \\ ${ }^{2}$ Department of Physics, International Centre of Quantum and Molecular Structures, and Materials Genome Institute, \\ Shanghai University, Shanghai 200444, China \\ ${ }^{3}$ Australian Nuclear Science and Technology Organisation, Locked Bag 2001, Kirrawee DC, NSW 2232, Australia \\ ${ }^{4}$ School of Chemistry and Molecular Bioscience, University of Wollongong, Wollongong, NSW 2522, Australia
}

a)Email: cheng@uow.edu.au

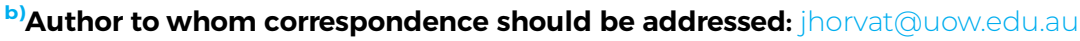

\begin{abstract}
Rare earth orthoferrites demonstrate great application potentials in spintronics and optical devices due to their multiferroic and magnetooptical properties. In $R \mathrm{FeO}_{3}$, magnetic $R^{3+}$ undergo a spontaneous spin reorientation in a temperature range determined by $R$ (rare earth), where the magnetic structure changes from $\Gamma_{2}$ to $\Gamma_{4}$. The $b$-axis component of their magnetic moment, $M_{b}$, is reported in numerous neutron diffraction studies to remain zero at all temperatures. More sensitive magnetometer measurements reveal a small non-zero $M_{b}$, which is minute above $\sim 200 \mathrm{~K} . M_{b}$ increases with cooling and reaches values of $\sim 10^{-3} \mu_{\mathrm{B}} /$ f.u. at temperatures within or below the spin reorientation temperatures. Our results can be explained by assuming the $\mathrm{Fe}^{3+}$ spins as the origin of non-zero $M_{b}$, while $R^{3+}$ spins suppress $M_{b}$. The representation analysis of point groups shows that non-zero $M_{b}$ is associated with a small admixture of the $\Gamma_{3}$ phase to $\Gamma_{2}$ or $\Gamma_{4}$. Such a mixing of the three magnetic phases requires at least a fourth order of the spin Hamiltonian for $R_{\mathrm{FeO}}$ to describe the non-zero $M_{b}$.
\end{abstract}

Published under license by AIP Publishing. https://doi.org/10.1063/1.5115518

\section{INTRODUCTION}

Rare earth orthoferrites, $R \mathrm{FeO}_{3}$, have been intensively studied since the 1960s due to their interesting magnetic properties. Research interest in these systems was recently renewed because some $\mathrm{RFeO}_{3}$ perovskites can exhibit magnetic field-induced ferroelectric polarization at a low temperature, thus becoming multiferroic. ${ }^{1-3}$ Furthermore, the ferroelectric polarization can be induced in these systems by strain breaking of space symmetry in their thin film form. ${ }^{4-6}$ Another interesting feature of $R \mathrm{FeO}_{3}$ is their ultra-fast spin dynamics. ${ }^{7,8}$ This makes them attractive candidates for the development of novel spintronic devices, where spin instead of the charge degree of freedom could be used in ultra-fast computers and terahertz sources. ${ }^{9-11}$ Their properties desirable for practical applications come at a cost: the magnetic interactions in these systems are very complex and are still not fully understood.
However, potential practical applications of these systems provide motivation to further elucidate their magnetic interactions, which this contribution attempts to do.

$R \mathrm{FeO}_{3}$ crystallize in an orthorhombic structure with space group Pbnm. Magnetic $\mathrm{Fe}^{3+}$ ions form a canted antiferromagnetic structure, with the Néel temperature between 650 and $700 \mathrm{~K}$ for the various rare earths, $R^{12-15}$ Their magnetic structure has been reported using the notation by Wollan and Koehler ${ }^{16}$ and Bertaut. ${ }^{17}$ Four $\mathrm{Fe}^{3+}$ ions are needed to describe this structure. At high temperatures, the magnetic structure of $\mathrm{Fe}^{3+}$ ions is $\Gamma_{4}=\left(G_{a}, A_{b}, F_{c}\right)$. The notations $G, F$, and $A$ represent the $(+-+-),(++++)$, and $(+--+)$ spin arrangements of the four $\mathrm{Fe}^{3+}$ ions, respectively. The indices $a, b$, and $c$ represent the crystalline directions corresponding to each of the spin arrangements. The main ordering at room temperature is antiferromagnetic of $G$-type along the crystalline $a$ axis. 
The Dzyaloshinskii-Moriya interaction is responsible for slight tilting of the $\mathrm{Fe}^{3+}$ spins. ${ }^{18-20}$ This tilting is symmetric into the crystalline $a$ axis, producing the ferromagnetic F-type ordering along the crystalline $c$ axis. It is antisymmetric along the crystalline $b$ axis, producing the antiferromagnetic $A$-type order. Therefore, there is a net ferromagnetic-type moment along the crystalline $c$ axis. $R \mathrm{FeO}_{3}$ systems with magnetic $R^{3+}$ undergo a spontaneous, gradual spin reorientation upon cooling within a temperature range that spans from several $\mathrm{K}$ to several tens of $\mathrm{K}$. For almost all $R \mathrm{FeO}$, the magnetic structure of $\mathrm{Fe}^{3+}$ ions below the spin reorientation temperature (SRT) is $\Gamma_{2}=\left(F_{a}, C_{b}, G_{c}\right)$, where $C_{b}$ represents the spin tilting arrangement $(++--)$ along the crystalline $b$ axis. Therefore, there is a net ferromagnetic-type moment along the $a$ axis below the spin reorientation temperature. The reorientation of the ferromagnetic spin component is reported to occur in the $(a, c)$ plane. $^{12,21-26}$ Consequently, a net magnetic moment along the crystalline $b$ axis is expected to be zero at all temperatures, which is also reported in numerous neutron diffraction studies of $R_{\mathrm{FeO}}$ magnetic structures. ${ }^{12,27-29}$ The magnetic moments of $R^{3+}$ ions order magnetically only below $\sim 5 \mathrm{~K}$. However, $R^{3+}$ ions become polarized by magnetically ordered $\mathrm{Fe}^{3+}$ ions at $T>5 \mathrm{~K}$, and they are in turn responsible for the spontaneous spin reorientation of the $\mathrm{Fe}^{3+}$ ions. ${ }^{12,14,22,26,30}$

$R \mathrm{FeO}_{3}$ have recently attracted much attention owing to their potential applications as spintronic and multiferroic materials. ${ }^{31,32}$ The focus of this research is mostly on the exact mechanisms of the spin reorientation, as well as on the details of their magnetic structure that can help explain the complex magnetic interactions and occurrence of multiferroicity in some of these systems. Neutron techniques have been the method of choice in many reports on magnetic properties of $R \mathrm{FeO}_{3}$, providing detailed information on magnetic interactions and structures for these systems. ${ }^{16,29,33,34}$ However, neutron techniques cannot provide as much sensitivity to the net magnetic moment as a vibrating sample magnetometer (VSM) can. Here, we report on accurate measurements of magnetic moments along the three crystalline directions for several types of $R \mathrm{FeO}_{3}$ single crystals. We show that there is a small but non-zero spontaneous net magnetic moment along the crystalline $b$ axis, which had not been picked up by the neutron techniques. Furthermore, the magnetism of $R^{3+}$ ions is not a prerequisite for this non-zero $b$ axis moment. Implications of the non-zero magnetic moment along the $b$ axis are discussed in terms of the representation theory of space groups. ${ }^{35}$ Our findings indicate the existence of another weak interaction in $\mathrm{RFeO}_{3}$ systems, which has not been accounted for in the current models.

\section{EXPERIMENTAL TECHNIQUES AND ANALYSIS PROCEDURE}

Single crystals of $\mathrm{NdFeO}_{3}, \mathrm{ErFeO}_{3}, \mathrm{HoFeO}_{3}$, and $\mathrm{YFeO}_{3}$ were measured. $\mathrm{YFeO}_{3}$ was the only system for which the rare earth ion, $\mathrm{Y}^{3+}$, was non-magnetic. ${ }^{12,13,36-38}$ It will help determine whether $\mathrm{Fe}^{3+}, R^{3+}$, or both contribute to the non-zero $b$ axis magnetic moment of $\mathrm{RFeO}_{3}$. The polarization of $\mathrm{Nd}^{3+}$ and $\mathrm{Er}^{3+}$ results in their net magnetic moment being the opposite to the net magnetic moment of $\mathrm{Fe}^{3+}$, while it is in the same direction as $\mathrm{Fe}^{3+}$ for $\mathrm{Ho}^{3+}$. The floating zone method was used for the growth of all crystals.
A mixture of $99.99 \%$ pure powders of $\mathrm{R}_{2} \mathrm{O}_{3}$ and $\mathrm{Fe}_{2} \mathrm{O}_{3}$ was used in a stoichiometric ratio. The powders were initially sintered at $1100{ }^{\circ} \mathrm{C}$ and then crushed and ground. Further sintering at $1350^{\circ} \mathrm{C}$ resulted in polycrystalline seed-rods. These rods were used for single crystal growth in a floating zone furnace. The furnace had four ellipsoidal mirrors, with four $1.0 \mathrm{~kW}$ halogen lamps as the heat sources. The rotation of the feed and seed shafts was in the opposite direction. The crystals were grown in 1 bar of oxygen in the vertical direction, from the bottom to the top. The melting zone traveling rate was in the range of $2.5-7.5 \mathrm{~mm} / \mathrm{h}$. Our single crystal samples were examined by $\mathrm{x}$-ray Laue photography to ascertain the crystallinity and crystallographic orientations. The backreflections of Laue XRD patterns (Fig. 1) confirmed an orthorhombically distorted perovskite structure with Pbnm symmetry for our single crystal samples, showing a high single crystallinity of the studied crystals with no evidence of twinning. The Laue XRD patterns also show that the cutting planes are perpendicular to $a, b$, and $c$ axes.

To further ascertain if there is any twinning in our crystals, single crystal $\mathrm{x}$-ray diffraction studies were performed at $294 \mathrm{~K}$ using monochromated Mo $\mathrm{K} \alpha$ radiation $(\lambda=0.71073 \AA)$ on samples of $\mathrm{ErFeO}_{3}$ using a Rigaku XtaLAB mini II Benchtop Single Crystal X-ray Diffractometer (SXRD).

This single wavelength technique is more sensitive for the detection of twins than Laue diffraction; however, it provides information only about a thin surface layer of the crystal. Small crystals $(<0.2 \mathrm{~mm}$ in size $)$ were cleaved from the surface of a large $\mathrm{ErFeO}_{3}$ crystal for these measurements. We determined that these small samples contained between $2 \%$ and $5 \%$ of twin components: $90^{\circ}$ around $001,180^{\circ}$ around 001,100 and 0.740 .670 .

In order to verify whether the results obtained on the cleaved samples were representative of the entire crystal, neutron diffraction measurements were performed using WOMBAT, the high intensity neutron diffractometer at the Australian Nuclear Science and Technology Organisation. Monochromatic neutrons with a wavelength of $1.5 \AA$ were used on the same $\mathrm{ErFeO}_{3}$ single crystal for which the magnetic measurements were performed earlier. Surprisingly, no evidence of twinning was observed. Taking into account the background noise, our detection limit for twin peaks was estimated as better than $1 \%$. It is likely that the cleaving of $<0.2 \mathrm{~mm}$ crystals for the XRD measurements created twins at the surface of cleaved-off crystallites, resulting in the twin structure described above. Nevertheless, we will assume that the twinning will contribute to the $b$-axis moment and this will be corrected for, as described in the Appendix.

Magnetic measurements were performed using the vibrating sample magnetometer (VSM) option of a Physical Property Measurement System (PPMS-9, Quantum Design). The frequency of VSM vibration was $40 \mathrm{~Hz}$ and amplitude was $2 \mathrm{~mm}$. The sweep rate of temperature was $2 \mathrm{~K} / \mathrm{min}$. The measurements of the temperature dependence of magnetic moment, $M(T)$, were conducted with the field-cooled-cooling (FCC) procedure. The value of magnetic moment was normalized to the formula unit (i.e., $R \mathrm{FeO}_{3}$ ), f.u., to allow a meaningful comparison between different $R \mathrm{FeO}_{3}$ systems.

Several possible artifacts had to be avoided to ascertain whether there is a spontaneous non-zero magnetic moment in the crystalline $b$ axis: the effect of magnetic domains, spin tilting by magnetic field $(H)$, sample misalignment in the sample holder, 

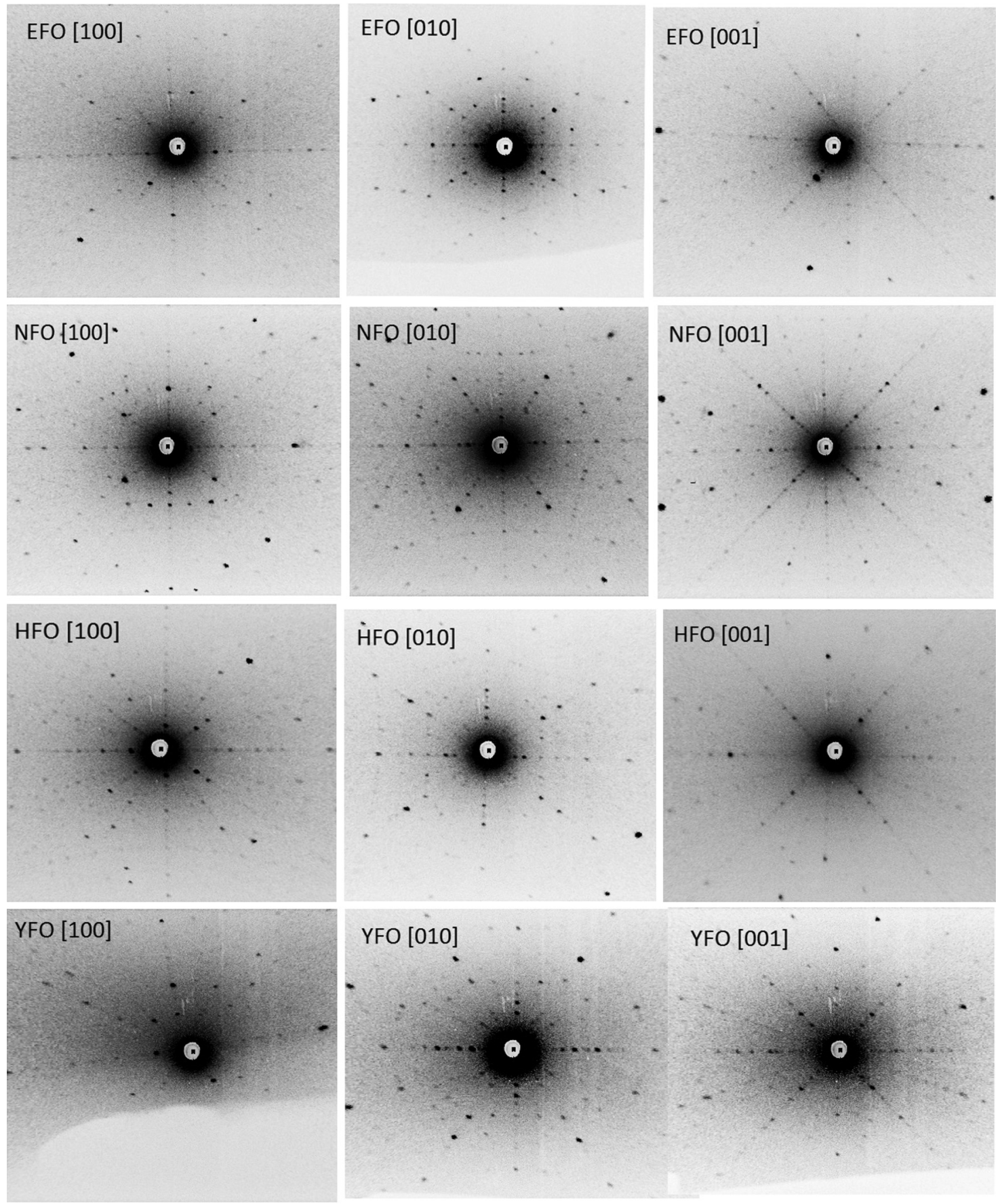

FIG. 1. X-ray Laue photographs for a-, $b$-, and $c$-crystalline axes, taken at room temperature for $R F \mathrm{O}_{3}$, where $\mathrm{R}=\mathrm{Nd}$, $\mathrm{Y}, \mathrm{Er}$, and $\mathrm{Ho}$.

irreversibilities associated with not heating the crystal to above the ordering temperatures of $\mathrm{Fe}^{3+}$ between different temperature scans and background moment due to the sample holder, and any small artifacts of the magnetometer.
Figure 2 displays magnetic hysteresis loops for $\mathrm{NdFeO}_{3}$ at different temperatures across the spin reorientation temperature (SRT) range. $M(H)$ loops measured along the $c$ axis are shown in Fig. 2(a), and those measured along the $a$ axis are shown in Fig. 2(b). 

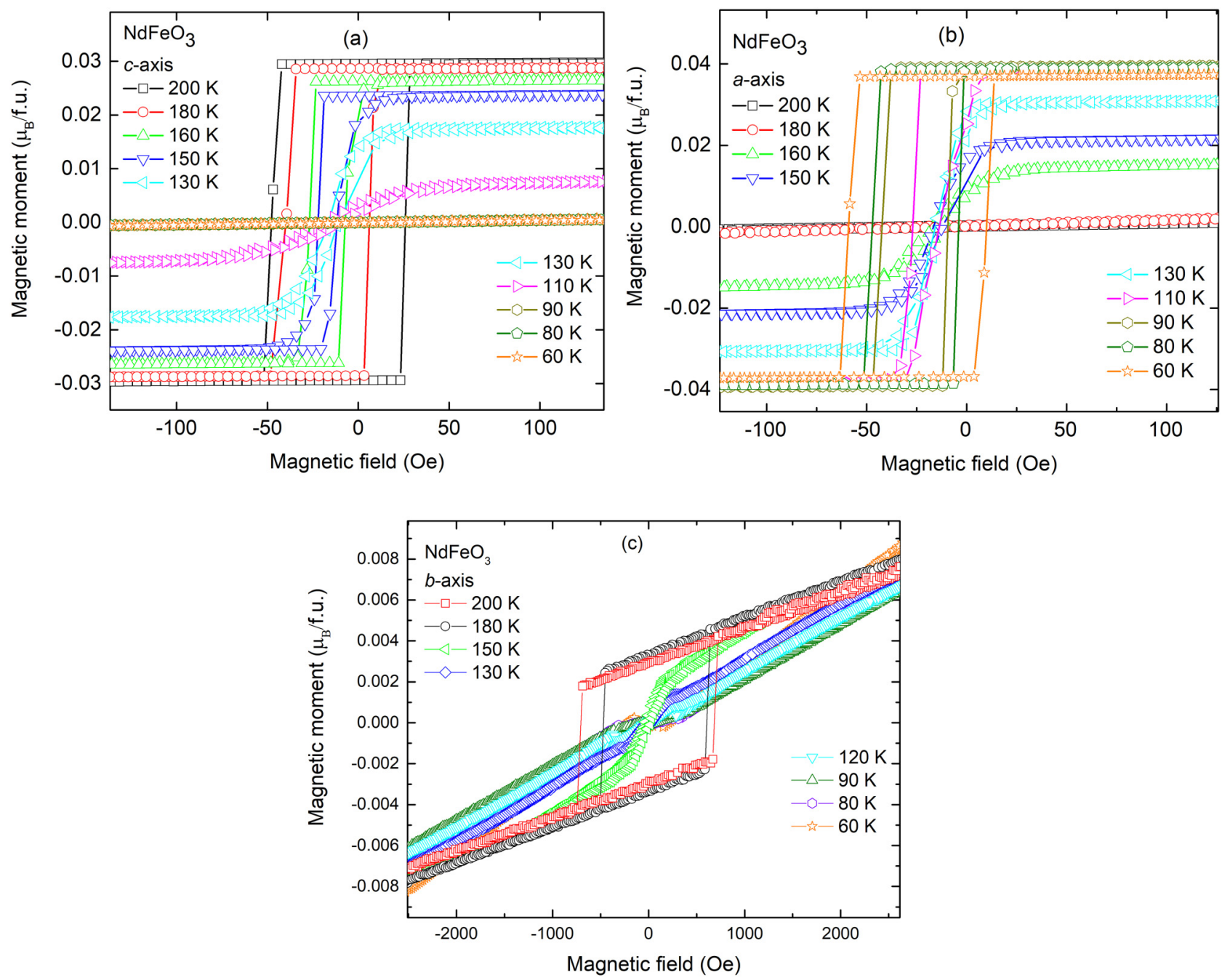

FIG. 2. Magnetic hysteresis loops for $\mathrm{NdFeO}_{3}$, measured along the crystalline $c$ axis (a), a axis (b), and $b$ axis (c). Nominal $b$-axis loops are dominated by the projections of $a$ - and $c$-axes moments.

There are three prominent features of the hysteresis loops: (a) abrupt changes in magnetization (rectangular shape) above the SRT range for the $c$ axis and below the SRT range for the $a$ axis, (b) a linear reversible change of $M$ below the SRT range for the $c$ axis and above the SRT range for the $a$ axis, and (c) S-shaped loops through the SRT range (170-100 K).

The values of the coercive field, $H_{c}$, were below $50 \mathrm{Oe}$, in agreement with other reports. ${ }^{39}$ Abrupt changes of the magnetization were assigned to the domain wall jumps, which represent extrinsic properties of the crystals. ${ }^{39,40}$ Their effects on $M(T)$ can be avoided by measuring at high enough fields, at which $M(H)$ is reversible, and there are no jumps in the hysteresis loops. Because of that, all $M(T)$ measurements were performed in the high-field, reversible regime. Typically, an applied field of $H>100$ Oe was needed in $M(T)$ measurements for $\mathrm{NdFeO}_{3}$ to avoid domain wall jumps at all temperatures. The value of the coercive field, $H_{c}$, for $\mathrm{ErFeO}_{3}$ was $70 \mathrm{Oe}$ above the SRT along the $c$ axis and below the SRT along the $a$ axis. Therefore, $M(T)$ measurements were also performed with an applied field of $\mathrm{H}>100 \mathrm{Oe}$. For the $\mathrm{HoFeO}_{3}$ crystal, $H_{c}$ was around 250 Oe when measuring $M_{c}$, so $M(T)$ measurements needed $H>300$ Oe to avoid domain wall artifacts. However, when measuring $\mathrm{HoFeO}_{3}$ along the $a$ axis, an anomaly in $M_{a}(T)$ was observed just below the spin reorientation temperature at elevated fields. This anomaly indicates the possible occurrence of a new field-induced magnetic phase at the elevated fields. As these elevated fields are needed to avoid the effects of magnetic domains on our measurements for this sample, $M_{a}(T)$ for $\mathrm{HoFeO}_{3}$ will not be considered in further analysis. Finally, for the $\mathrm{YFeO}_{3}$ sample, $H_{c}$ had a high value around 2000 Oe. Therefore, $H>2000$ Oe was needed to avoid the domain wall artifacts. Hysteresis loops 
measured along the nominal $b$ axis are contributed mainly by $a$ - and $c$-axes moments because of small sample misalignment in the magnetometer [Fig. 2(c)].

The linear, reversible part of the hysteresis loops indicates how much the spins are tilted by the applied magnetic field through the temperature-dependent value of magnetic susceptibility. Tilting of the spins by a magnetic field can result in values of magnetic moment comparable to the magnetic moment along the crystalline $b$ axis $\left(M_{b}\right)$, and the effect of the tilting needs to be eliminated from our measurements. To obtain the spontaneous magnetic moment, $M(T)$ measurements were performed at different values of the applied field $(H)$ for each crystalline direction with values of $H$ high enough to eliminate the magnetic domains in the crystals. Care was taken not to apply a too high field, however, which could change the underlying magnetic structure so that the extrapolation to $H=0$ would not be meaningful (like in the case of $M_{a}$ for $\mathrm{HoFeO}_{3}$ ). For each $T$, the values of the magnetic moment measured at different fields were then extrapolated to $H=0$. This procedure was used for all three crystalline directions to avoid the spin tilting effects at temperatures where $M_{a}$ and $M_{c}$ become small. Figure 3 illustrates this procedure for the case of $\mathrm{NdFeO}_{3}$. For each temperature, the value of magnetization measured at different fields was fitted with a straight line and extrapolated to $H=0$.

When measuring along the nominal $b$ axis, only a small projection of the external field is aligned with $a$ and $c$ axes due to small sample misalignment. Because $M_{a}$ and $M_{c}$ are much larger than $M_{b}, M_{a}$ and $M_{c}$ determine the domain structure of the sample. The sample was, therefore, pre-magnetized with a large field ( $\sim 5000$ Oe), ensuring that the sample is in the mono-domain state represented by the linear $M(H)$ regime in Fig. 2 before
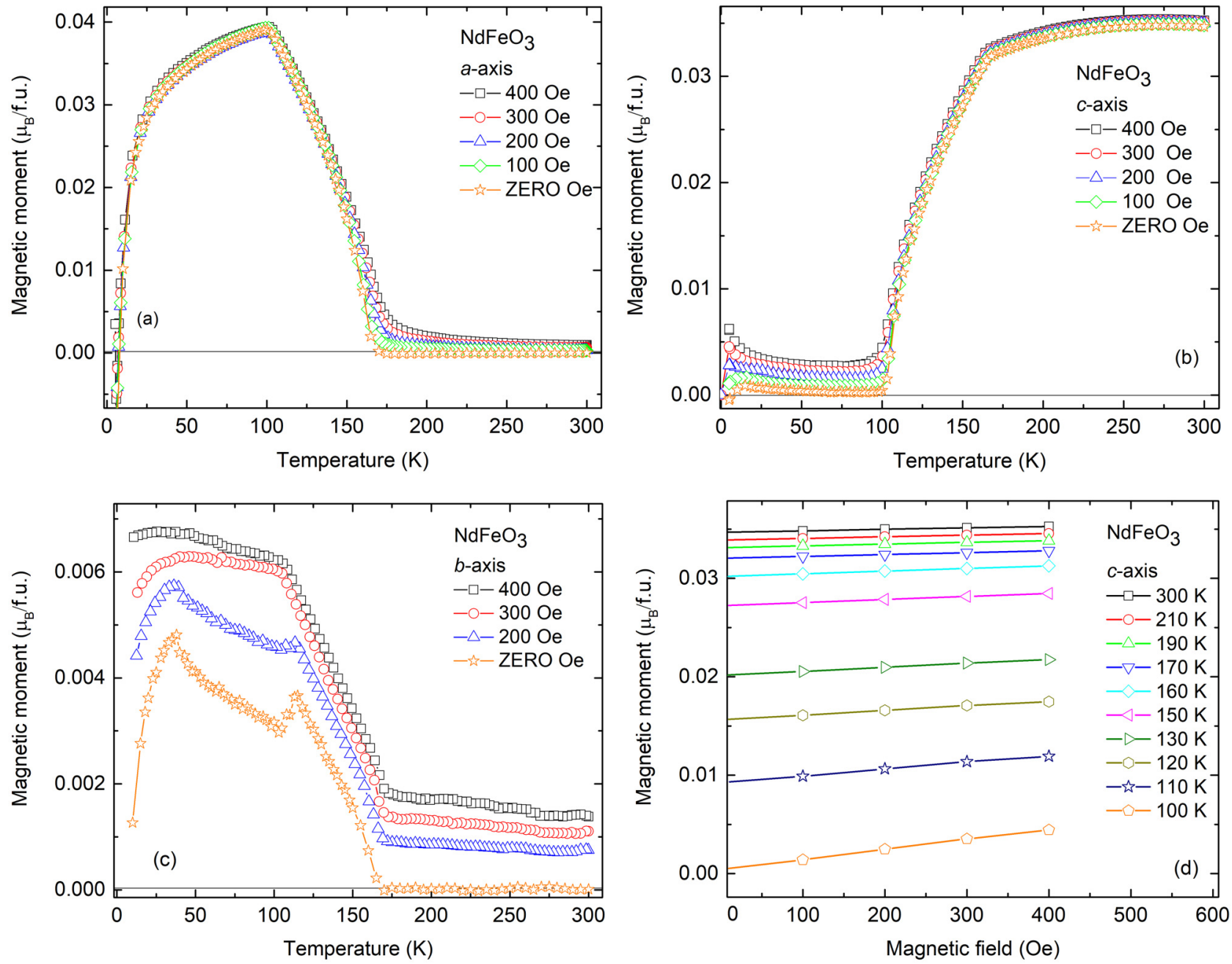

FIG. 3. Extrapolation of $M(T)$ measured at elevated fields to $\mathrm{H}=0$ for $\mathrm{NdFeO}_{3}$ in the direction of the crystalline: (a) a axis, (b) $c$ axis, and (c) $b$ axis. (d) Linear extrapolation of measured magnetic moment to $\mathrm{H}=0$ for $\mathrm{NdFeO}_{3}$ crystal. 
measuring $M_{b}(T)$ at smaller fields. In this way, consistency between all measurements was achieved.

As values of $M_{b}$ are expected to be zero (or very small), while $M_{a}$ and $M_{c}$ are much larger than $M_{b}$, any sample misalignment in the magnetometer will result in the projection of $M_{a}$ and $M_{c}$ onto the nominal $b$ axis that is comparable to any real non-zero $M_{b}$. Furthermore, any twinning of the crystal that could provide a non-zero projection of $M_{a}$ and $M_{c}$ of the twinned volume onto the crystalline $b$ axis would also give an artifact of non-zero $M_{b}$. To avoid both these artifacts in our experiment, temperature dependence of the magnetic moment was compared along each of the crystalline axes. If $M_{b}$ occurs through a projection of $M_{a}$ and $M_{c}$ onto the nominal $b$ axis of the measurement, $M_{b}$ should follow the temperature dependence of $M_{a}$ and $M_{c}$, i.e., the linear combination of the two. If, however, a different temperature dependence of the measured $M_{b}$ is obtained, non-zero $M_{b}$ must exist. This procedure is described in more detail in the Appendix, which also shows that the correction for the sample misalignment and twinning has the same mathematical form.

In our measurements, crystals were measured at temperatures up to $300 \mathrm{~K}$, which is well below the ordering temperature of $\mathrm{Fe}^{3+}$ spins. This could lead to artifacts associated with irreversibilities of the $\mathrm{Fe}^{3+}$ system and polarization of $\mathrm{R}^{3+}$ ions by ordered $\mathrm{Fe}^{3+}$. To avoid these artifacts, sample demagnetization from above $1 \mathrm{~T}$ in field cycles with amplitude gradually decreasing to zero helped maintain the reproducibility of the measurements. If there were any unusual non-reproducible jumps or steps obtained in $M(T)$, the whole measurement was discarded.

To eliminate the magnetic moment of the sample holder and any other artifacts of the magnetometer, we performed $M(T)$ measurements of the paramagnetic $\mathrm{Dy}_{2} \mathrm{O}_{3}$ sample in the same way and with the same sample holder as for $M(T)$ measurements of $\mathrm{RFeO}_{3}$ samples at the same magnetic fields. Extrapolation of $M(T)$ for $\mathrm{Dy}_{2} \mathrm{O}_{3}$ to $H=0$ gave the background $M(T)$ that was subtracted from the measurements for $R_{\mathrm{FeO}}$. This correction was much smaller than typical values of $M_{b}$ extrapolated to $H=0$ and corrected for the sample misalignment and twinning effects.

\section{RESULTS AND DISCUSSION}

The temperature dependence of the magnetization extrapolated to $H=0$ along the three crystalline axes is shown in Fig. 4 for all $R \mathrm{FeO}_{3}$ samples measured. For crystals with magnetic rare earth, $R=\mathrm{Nd}$, Er, and $\mathrm{Ho}$, the magnetization at room temperature lies along the crystalline $c$ axis. $M_{c}$ slowly decreases with cooling for $R=\mathrm{Nd}^{3+}$ and $\mathrm{Er}^{3+}$, because the average moment of polarized $R^{3+}$ ions is oriented in the opposite direction to the ferromagnetic spin component of magnetically ordered $\mathrm{Fe}^{3+}$ ions. ${ }^{41-44}$ As the polarization of $R^{3+}$ strengthens with cooling, the net magnetic moment decreases. For $R=\mathrm{Ho}, M_{c}$ increases with cooling because the polarized moment of $\mathrm{Ho}^{3+}$ is oriented in the same direction as the ferromagnetic component of the ordered $\mathrm{Fe}^{3+}$ ions. ${ }^{41}$ Spontaneous spin reorientation occurs continuously within a temperature range specific to each $R$, which we denote as the SRT range. In the SRT range, $M_{c}$ decreases with cooling and $M_{a}$ at the same time increases. The range of SRT is $100-170 \mathrm{~K}, 85-95 \mathrm{~K}$, and $49-58 \mathrm{~K}$ for $R=\mathrm{Nd}$, Er, and Ho, respectively. The reorientation of the ferromagnetic spin component is commonly described as occurring in the $(a, c)$ crystalline plane. ${ }^{12,21,22} M_{a}$ of $\mathrm{Fe}^{3+}$ is expected to be zero at $300 \mathrm{~K}$ and $M_{c}$ is expected to be zero below SRT. A deviation in measured $M_{a}$ and $M_{c}$ from zero is often due to the sample misalignment in the magnetometer so that the projection of $M_{c}$ appears in the measurements of $M_{a}$ above SRT or the projection of $M_{a}$ appears in the measurements of $M_{c}$ below SRT. The deviation from zero can also be caused by the polarization of $R^{3+}$ ions by magnetically ordered $\mathrm{Fe}^{3+}$ ions. The sample misalignment effects can be corrected for, however, only the measurements with a near-zero $M_{a}$ at $300 \mathrm{~K}$ and near-zero $M_{c}$ just below SRT were used for the extraction of $M_{b}$, for which there was no need for further corrections. $\mathrm{ErFeO}_{3}$ additionally displays an abrupt magnetization reversal from negative to positive values on cooling at temperatures well below SRT (below $50 \mathrm{~K}$ ). This abrupt magnetization reversal at field-dependent temperatures renders our procedure for the magnetization extrapolation to $H=0$ unsuitable at these temperatures, and for this reason, the $M$ (T) curves of $\mathrm{ErFeO}_{3}$ will not be considered in the analysis of $M_{b}$ (T) below $45 \mathrm{~K}$.

The gradual change of the magnetization in the SRT region does not suffer from such issues. The magnetization reversals also occur for $\mathrm{NdFeO}_{3}$ but below $10 \mathrm{~K}$ in our samples, and they are not shown in Fig. 4. $M_{b}$ is small but non-zero in our measurements for all the samples, especially in the SRT region. We will show later that the obtained non-zero $M_{b}(T)$ cannot be interpreted by the sample misalignment in the magnetometer or crystal twinning, but it is a real phenomenon. Measurements of $\mathrm{YFeO}_{3}$ are shown in Fig. 4(d). The net magnetization of $\mathrm{YFeO}_{3}$ is oriented along the crystalline $c$ axis at all temperatures, without spontaneous spin reorientation. This magnetization is contributed by $\mathrm{Fe}^{3+}$ only, as $\mathrm{Y}^{3+}$ is non-magnetic in $\mathrm{YFeO}_{3} \cdot{ }^{38}$ Non-zero $M_{a}(T)$ and $M_{b}(T)$ are also measured for $\mathrm{YFeO}_{3}$.

The temperature dependence of the measured $M_{b}$ extrapolated to $H=0$ is shown in Fig. 5. $M_{b}$ is apparently non-zero for all samples measured. To ascertain if this non-zero $M_{b}$ was obtained because of slight sample misalignment in the sample holder and/or twinning effects, we attempted to fit it with a linear combination of $M_{a}$ and $M_{c}: M_{b, f i t}=\alpha M_{c}+\beta M_{a}$. As shown in the Appendix, agreement between $M_{b, f i t}(T)$ and measured magnetic moment along the $b$ axis would signify that the real $M_{b}(T)$ is zero. Fitting parameters $\alpha$ and $\beta$ contain the cosines of the misalignment angles, accounting for the projections of $M_{a}$ and $M_{c}$ onto the magnetometer axis, together with the Euler angles representing the projections of the twinned sample volumes (Appendix). Initial values of $\alpha$ and $\beta$ were obtained from the experiment. The value of $\alpha$ was obtained as $M_{b} / M_{\mathrm{c}}$ at $300 \mathrm{~K}$ for the samples with magnetic $R^{3+}$, where $M_{a} \approx 0$. The value of $\beta$ was obtained as $M_{b} / M_{a}$ at a temperature just below the SRT region, where $M_{c} \approx 0$. Further refinement of the values for $\alpha$ and $\beta$ was performed to further improve the agreement between $M_{b, f i t}(T)$ and the nominal measured $M_{b}(T)$. As seen in Fig. 5, the measured $M_{b}$ is rather different to the best data-matching $M_{b \text {,fit }}$. For $\mathrm{HoFeO}_{3}$, only the data above spin reorientation are shown because $M_{a}(T)$ could not be obtained at lower temperatures, as described above. Therefore, the measured non-zero $M_{b}$ is not an artifact of crystal twinning or the sample misalignment in the magnetometer, but an intrinsic property of the samples measured. 

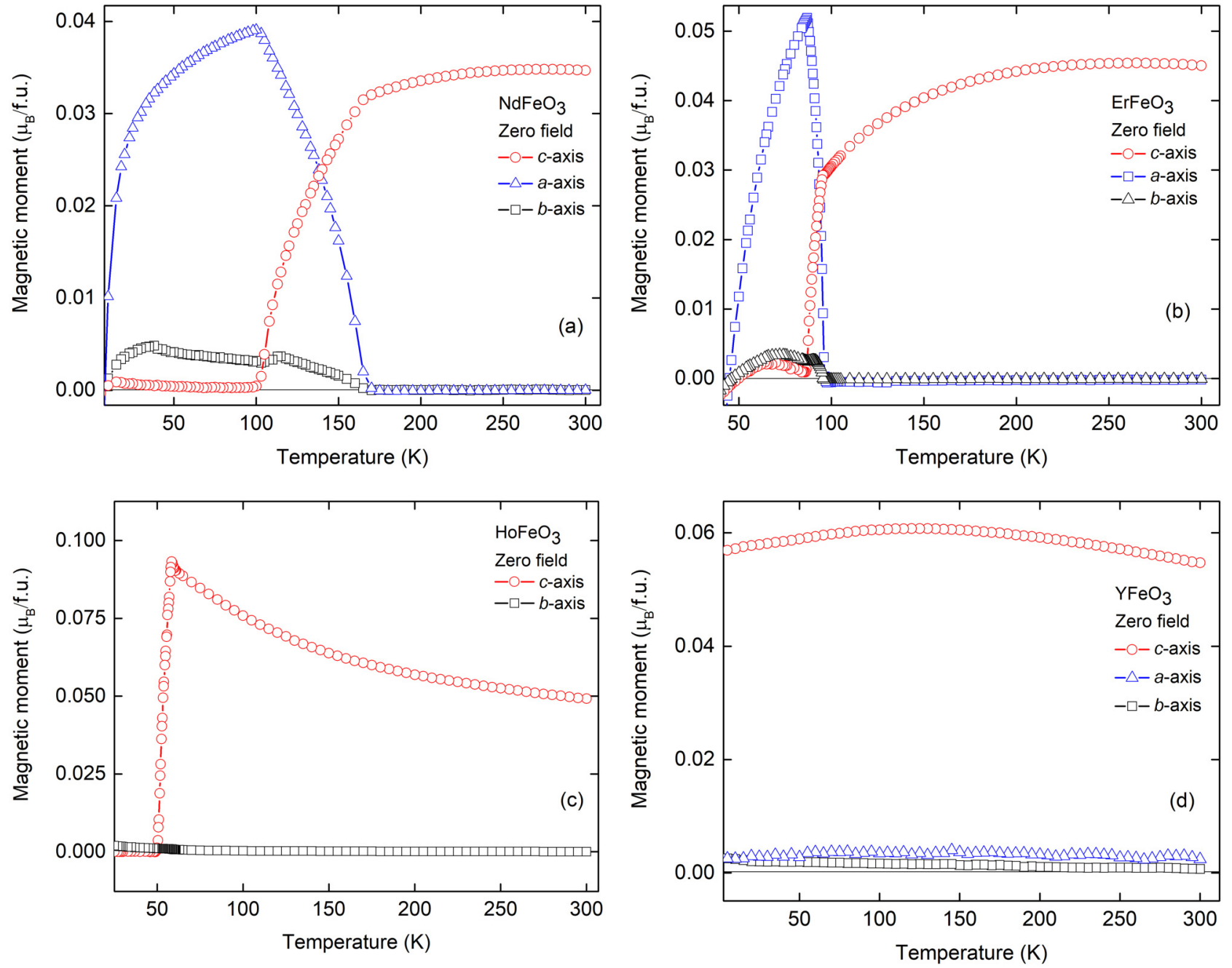

FIG. 4. Temperature dependence of magnetic moment measured along crystalline $a, b$, and $c$ axes in extrapolation to $\mathrm{H}=0$ : (a) $\mathrm{NdFeO}_{3}$, (b) $\mathrm{ErFeO}_{3}$, (c) $\mathrm{HoFeO}_{3}$, and (d) $\mathrm{YFeO}_{3}$.

Qualitatively, the same argument was obtained by using the data measured at a non-zero field, i.e., not extrapolated to $H=0$. Subtracting the $M_{b, f i t}(T)$ from the measured $M_{b}(T)$ provides the temperature dependence of intrinsic $M_{b}$.

For $\mathrm{YFeO}_{3}, M_{a}(T)$ can be fitted well with the projection of $M_{c}$ (T) onto the magnetometer axis and it occurs in our measurements because of the sample misalignment in the magnetometer by $5^{\circ}$ when measuring $M_{a}(T) . M_{b}(T)$ cannot be fitted by linear combinations of $M_{c}(T)$ and $M_{a}(T)$, just as for the other crystals. As $\mathrm{YFeO}_{3}$ does not undergo spin reorientation, it is obvious for this sample that $M_{b}(T)$ would have to be proportional to $M_{c}(T)$ if sample misorientation and twinning were responsible for the obtained non-zero $M_{b}$. Our measurements unquestionably exclude this possibility (Fig. 5), indicating that $\mathrm{YFeO}_{3}$ has intrinsic non-zero $M_{b}(T)$.
The final $M_{b}(T)$, after all of the corrections described above, is shown in Fig. 6. By obtaining the $M_{b}(T)$ through subtraction of the $M_{b, f i t}$ from the measured $M_{b}$, it is assumed that the net value of the intrinsic $M_{b}$ is zero at room temperature. This assumption can be justified for systems with magnetic $R^{3+}$ by the negligible experimental value of $M_{b}$ and its weak temperature dependence, if any, for $T>200 \mathrm{~K}$. Furthermore, the measured $M_{b} / M_{c}$ is temperature independent above $200 \mathrm{~K}$ for $\mathrm{NdFeO}_{3}$ and $\mathrm{ErFeO}_{3}$, suggesting that the measured $M_{b}$ is merely a projection of $M_{c}$ onto the magnetometer axis at these temperatures. Without this assumption, the values of $M_{b}$ for each $R_{\mathrm{FeO}}$ would be given up to an unknown additive constant above SRT. The value of $M_{b}$ for $R \mathrm{FeO}_{3}$ with magnetic $R^{3+}$ is the largest at the peak within the SRT region and also at a lower temperature. This would indicate that the polarization of $R^{3+}$ ions is responsible for the non-zero $M_{b}$, as the polarization is strongest 

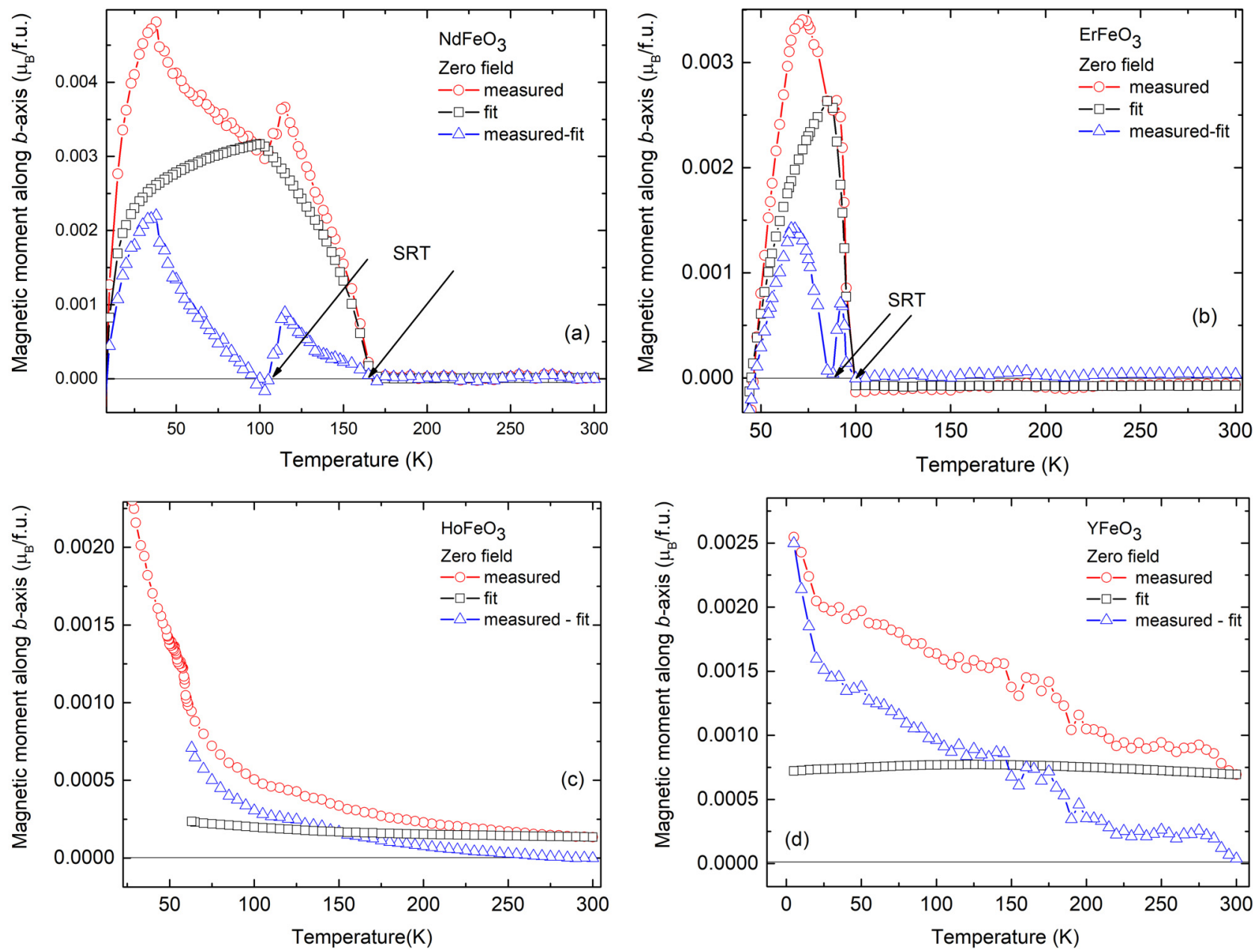

FIG. 5. The temperature dependence of the measured, fitted, and intrinsic $M_{b}$ (i.e., measured $M_{b}$ - fit to $M_{b}$ ) for all $R \mathrm{FeO}_{3}$ measured: (a) $\mathrm{NdFeO}_{3}$, (b) $\mathrm{ErFeO}_{3}$, (c) $\mathrm{HoFeO}_{3}$, and (d) $\mathrm{YFeO}_{3}$.

at low temperatures. Measurements on $\mathrm{YFeO}_{3}$, for which $\mathrm{Y}^{3+}$ is non-magnetic, help assess the validity of this hypothesis. Figures 5 and 6 show that the value of $M_{b}$ is non-zero for $\mathrm{YFeO}_{3}$, as well, but the temperature dependence is qualitatively quite different than for the other $\mathrm{RFeO}_{3}$. The value of $M_{b}$ can no longer be assumed to be zero at room temperature for $\mathrm{YFeO}_{3}$ because $M_{b} / M_{c}$ is not temperature independent. Therefore, the absolute values of $M_{b}$ cannot be determined by our method for $\mathrm{YFeO}_{3}$, and Figs. 5(d) and 6 show its temperature dependence up to an additive constant. The remarkable difference in $M_{b}(T)$ between $\mathrm{YFeO}_{3}$ and other $R \mathrm{FeO}_{3}$ crystals with magnetic $R^{3+}$, as well as the non-zero $M_{b}(T)$ of $\mathrm{YFeO}_{3}$, together imply that for magnetic $R^{3+}$, both $R^{3+}$ and $\mathrm{Fe}^{3+}$ contribute to the net value of $M_{b}$.

Figure 6 shows that $M_{b}$ is close to zero at room temperature for all $R \mathrm{FeO}_{3}$ with magnetic $R^{3+}$. For $R=\mathrm{Nd}, \mathrm{Er}$, and $M_{b}$ remains zero upon cooling above the SRT region and then increases strongly with further cooling below the onset temperature of spin reorientation, reaching a positive maximum within the SRT region. It reaches another maximum below the SRT region. For $\mathrm{HoFeO}_{3}$, for which the net moments of $R^{3+}$ and $\mathrm{Fe}^{3+}$ are in the same direction above SRT, $M_{b}$ increases with cooling below $\sim 250 \mathrm{~K}$, even above the SRT range. $M_{b}$ cannot be obtained below SRT with the methods employed here for $\mathrm{HoFeO}_{3}$. For $\mathrm{YFeO}_{3}$, $M_{b}$ just increases monotonously with cooling. We emphasize that our procedure of fitting the measured $M_{b}(T)$ with a linear combination of $M_{a}(T)$ and $M_{c}(T)$ and then taking the intrinsic $M_{b}$ (T) to be the difference between the measured $M_{b}(T)$ and this fit can result in a distortion of the thus obtained $M_{b}(T)$. This is because we are first assuming that our measured $M_{b}(T)$ might consist only of the projections of $M_{a}$ and $M_{c}$ on the magnetometer axis when measuring $M_{b}(T)$. With this assumption, we take for the measured $M_{b}$ that $M_{b}=\beta M_{a}$ at the temperature just below 


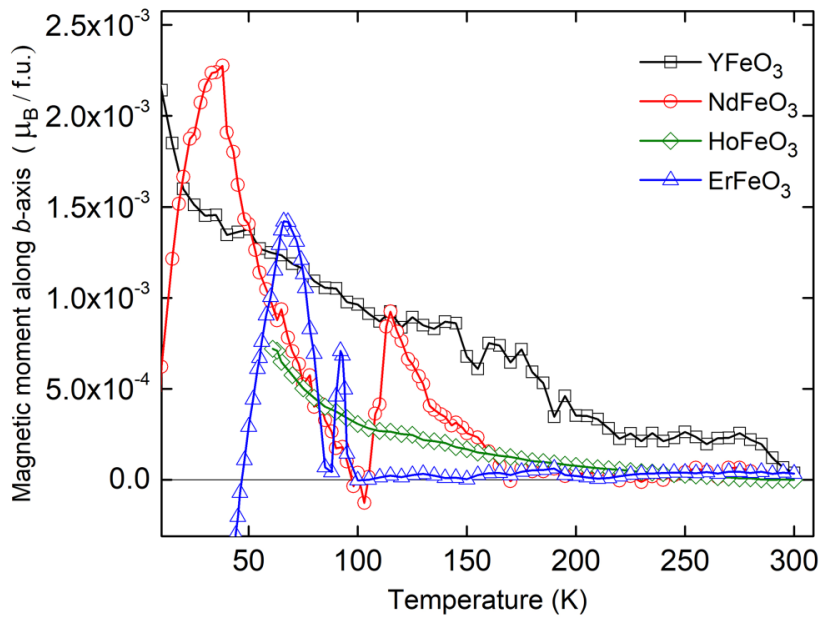

FIG. 6. Comparison of the temperature dependence of $M_{b}$ extrapolated to $H=0$ for all the samples measured, after correcting for the sample misalignments, twinning, and instrumental background. Magnetic moment was normalized to the formula unit (f.u.) for each system. The spin reorientation occurs between: 100 and $170 \mathrm{~K}$ for $\mathrm{NdFeO}_{3}, 89$ and $95 \mathrm{~K}$ for $\mathrm{ErFeO}_{3}$, and 49 and $58 \mathrm{~K}$ for $\mathrm{HoFeO}_{3}$.

the spin reorientation, which fixes the value of the "real" $M_{b}$ to zero at this temperature. However, it is possible that the intrinsic $M_{b}$ at the temperature just below the spin reorientation is non-zero, which would change the slope of $M_{b}(T)$ in Fig. 6 . Despite this ambiguity, our procedure still shows that the intrinsic $M_{b}(T)$ is non-zero in a broad temperature range for $R_{\mathrm{FeO}}$ and it provides $M_{b}(T)$, defined up to an unknown additive linear function of $T$.

Figure 6 shows that $\mathrm{YFeO}_{3}$ has the largest $M_{b}$, and consequently, $\mathrm{Fe}^{3+}$ ions are the main contributors to $M_{b}$ above SRT. We note that $M_{b}$ was arbitrarily assumed to be zero for $\mathrm{YFeO}_{3}$ at $300 \mathrm{~K}$ through our procedure correcting for the sample misalignment and crystal twinning. Because $M_{b}(T)$ for $\mathrm{YFeO}_{3}$ is a monotonously decreasing function of $\mathrm{T}$ and $\mathrm{YFeO}_{3}$ has no known magnetic phase transition in this temperature range, it is unlikely it would become negative above $300 \mathrm{~K}$. No spin reorientation is reported for $\mathrm{YFeO}_{3}$. Consequently, it would make sense to assume that $M_{b}$ should reach zero at the Néel temperature for $\mathrm{YFeO}_{3}$, i.e., at $\sim 645 \mathrm{~K}^{22,37,45}$ and not at $300 \mathrm{~K}$. This means that the true values of $M_{b}$ for $\mathrm{YFeO}_{3}$ could be larger than shown in Fig. 6.

These measurements of $M_{b}$ can be explained by assuming that the magnetic moment of $R^{3+}$ strongly suppresses $M_{b}$ at temperatures above $\sim 200 \mathrm{~K}$, giving $M_{b} \approx 0$. This suppression appears to be significantly disrupted in the SRT region and also at temperatures well below the SRT range, where the polarization of $R^{3+}$ is strong. The polarization of $R^{3+}$ was shown to change substantially through the SRT region, which supports our argument. ${ }^{46}$ Therefore, the polarization of $R^{3+}$ spins plays a significant role in $M_{b}$. While it may sound odd that $R^{3+}$ spins would have such a strong effect at temperatures where they are only weakly polarized, it is worth mentioning that these unpolarized spins are also responsible for the occurrence of spontaneous spin reorientation in these systems. Nevertheless, these arguments should be further tested by measuring other $R \mathrm{FeO}_{3}$ with non-magnetic $R^{3+}$.

We note that the obtained $M_{b}(T)$ is at odds with the idea of $M_{b}$ being an artifact of crystal twinning. XRD measurements on small cleaved crystals revealed the presence of $180^{\circ}$ and $90^{\circ}$ twins. The $180^{\circ}$ twins around the principal axes merely change the sign of the crystalline axis without affecting the magnetic moment. For twinning that contributes to $M_{b}, a$ and $b$ axes are exchanged in a limited sample volume, i.e., they are $90^{\circ}$ twins. This means that a small $M_{a}(T)$ contribution by the twinned volume would be observed below SRT when measuring along the crystalline $b$ axis. However, the obtained $M_{b}$ has a rather different temperature dependence than $M_{a}$. Furthermore, the direction of the magnetic moment may vary randomly within a family of $90^{\circ}$ twins, as magnetic anisotropy energy is the same for + and - direction of the crystalline axis. Therefore, with statistically significant distribution of the twins, their magnetic moment would add up to $\sim 0$, similar to a system of magnetic domains. Finally, neutron diffraction experiments show no presence of the twins in a large $\mathrm{ErFeO}_{3}$ crystal on which the magnetic measurements were performed, which further implies the effect of twinning on the measured $M_{b}$ would be insignificant.

The literature overwhelmingly reports $M_{b}$ of $R \mathrm{FeO}_{3}$ to be zero, mostly on the basis of neutron techniques. However, the antiferromagnetic axis tilts toward and away from the $b$ axis with a temperature change. For example, a neutron powder diffraction study of $\mathrm{ErFeO}_{3}$ reveals an antiferromagnetic spin component of $\mathrm{Fe}^{3+}$ along the $b$ axis of $\sim 1 \mu_{\mathrm{B}} / \mathrm{f}$.u. at $4 \mathrm{~K}{ }^{47}$ Its value decreases with $T$ and is no longer detectable above $8 \mathrm{~K}$. A similar trend is reported for $\mathrm{Er}^{3+}$ spins. Magnetic resonance, ${ }^{48}$ magnetostriction, ${ }^{49}$ and Mössbauer studies of $\mathrm{HoFeO}_{3}$ crystals show that the antiferromagnetic vector, $G$, of $\mathrm{Fe}^{3+}$ is tilted by up to $20^{\circ}$ toward the crystallographic $b$ axis in its SRT region and $\sim 10^{\circ}$ outside this region. These reports show that the antiferromagnetically coupled spin components in $R \mathrm{FeO}_{3}$ spontaneously rotate out of the $(a, c)$ plane with a temperature change. These spin components do not contribute to the net magnetic moment along the crystalline $b$ axis. However, any disruption of the symmetry within this antiferromagnetic coupling, affected by the change of complex magnetic anisotropies in $R \mathrm{FeO}_{3}$ with $T$, would effectively result in a ferromagnetic component along the $b$ axis.

The representation analysis of space groups ${ }^{17}$ gives all possible magnetic structures that are allowed on the basis of the symmetry of a particular crystal. The crystal structure of $R^{2} \mathrm{FeO}_{3}$ can be described by the space group Pbnm. All magnetic reflections can be indexed by the crystallographic system with the propagation vector $\mathbf{k}=0 .{ }^{47}$ The irreducible representations $\Gamma_{1}-\Gamma_{8}$ obtained in this analysis describe the allowed magnetic structures for $\mathrm{Pbnm}$ space group on the basis of symmetry considerations, as given in Table I. ${ }^{17}$ The symmetry-allowed representations $\Gamma_{5}-\Gamma_{8}$ are not realized for $\mathrm{Fe}^{3+} . \Gamma_{1}$ consists only of antiferromagnetic components $A, G$, and $C$ and, therefore, does not describe the spin structure of those of $\mathrm{RFeO}_{3}$, for which experiments reveal ferromagnetic components. $\Gamma_{1}$ is reported to co-exist with $\Gamma_{2}$ in the SRT region of $\mathrm{HoFeO}_{3}{ }^{48}$ or on its own below the SRT region of $\mathrm{DyFeO}_{3}$; $^{50}$ 
TABLE I. Allowed magnetic structures for Pbnm space group, obtained from the representation analysis of space groups. ${ }^{17}$ The subscripts $a, b$, and $c$ refer to crystalline $a, b$, and $c$ axes, respectively. The spin arrangement of the four $\mathrm{Fe}^{3+}$ or the four $R^{3+}$ sites in the unit cell is described by the base vectors: $A=(+,-,-,+)$, $G=(+,-,+,-), C=(+,+,-,-)$, and $F=(+,+,+,+)$.

\begin{tabular}{lcc}
\hline \hline $\begin{array}{l}\text { Irreducible } \\
\text { representation }\end{array}$ & $\begin{array}{c}\text { Structure for } \\
\mathrm{Fe}^{3+} \text { sites }\end{array}$ & $\begin{array}{c}\text { Structure for } \\
R^{3+} \text { sites }\end{array}$ \\
\hline$\Gamma_{1}$ & $A_{a} G_{b} C_{c}$ & $C_{c}$ \\
$\Gamma_{2}$ & $F_{a} C_{b} G_{c}$ & $F_{a} C_{b}$ \\
$\Gamma_{3}$ & $C_{a} F_{b} A_{c}$ & $C_{a} F_{b}$ \\
$\Gamma_{4}$ & $G_{a} A_{b} F_{c}$ & $F_{c}$ \\
$\Gamma_{5}$ & $\ldots$ & $G_{a} A_{b}$ \\
$\Gamma_{6}$ & $\ldots$ & $A_{c}$ \\
$\Gamma_{7}$ & $\ldots$ & $G_{c}$ \\
$\Gamma_{8}$ & $\ldots$ & $A_{a} G_{b}$ \\
\hline \hline
\end{tabular}

however, because $M_{b}$ is considered to be zero for $\mathrm{RFeO}_{3}$ systems, their magnetic structure is usually described by $\Gamma_{2}$ and $\Gamma_{4}$, which do not have ferromagnetic components along the crystalline $b$ axis (Table I).

Our measurements, however, give non-zero $M_{b}$ over a broad temperature range (Fig. 6). The only irreducible representation that allows the net non-zero $M_{b}$ is $\Gamma_{3}$ (Table I), for which both $\mathrm{Fe}^{3+}$ and $R^{3+}$ sites have a ferromagnetic component along the crystalline $b$ axis. This is in excellent agreement with our measurements for $R \mathrm{FeO}_{3}$ with magnetic $R^{3+}$ and $\mathrm{YFeO}_{3}$ with non-magnetic $\mathrm{R}^{3+}$ (Fig. 6), which can be explained only if both $\mathrm{Fe}^{3+}$ and $R^{3+}$ have a magnetic component along the crystalline $b$ axis. The $b$ axis ferromagnetic component of $R^{3+}$ seems to be oriented in the opposite direction to $\mathrm{Fe}^{3+}$, fully compensating the $M_{b}$ contributed by $\mathrm{Fe}^{3+}$ above $\sim 200 \mathrm{~K}$ (Fig. 6). Therefore, our measurements imply that there is a small admixture of the $\Gamma_{3}$ structure to either $\Gamma_{2}$ or $\Gamma_{4}$ structure outside the SRT region. Within the SRT region, $\Gamma_{2}, \Gamma_{3}$, and $\Gamma_{4}$ structures co-exist. This is in agreement with the symmetry considerations since any spin system can belong to up to three irreducible representations. ${ }^{17}$ This $\Gamma_{3}$ occurs spontaneously in $R \mathrm{FeO}_{3}$, as opposed to the field-induced $\Gamma_{3}$ structure reported earlier for $\mathrm{HoFeO}_{3}{ }^{51}$

The observation of non-zero $M_{b}(T)$ also points to the type of the spin Hamiltonian required for the description of the corresponding magnetic structure. The representation analysis shows that for $R \mathrm{FeO}_{3}$ systems with non-magnetic $R^{3+}$, fourth or even a higher order of spin Hamiltonian is required if the spin components do not belong to the same irreducible presentation at a given temperature. ${ }^{17}$ For $\mathrm{RFeO}_{3}$ with magnetic $R^{3+}$, however, the Hamiltonian does not necessarily have to be of the order larger than two if spins belong to more than one irreducible representation. Therefore, the appearance of non-zero $M_{b}(T)$ associated with an admixture of the $\Gamma_{3}$ phase for $\mathrm{YFeO}_{3}$ requires the Hamiltonian of the order four or more in the spins, to account for the observed non-zero $M_{b}(T)$. Even though the representation analysis does not require such a Hamiltonian for $R_{\mathrm{FeO}}$ with magnetic $R^{3+}$, it also does not exclude it either, and the same may also be true for them, at least below $200 \mathrm{~K}$.

\section{CONCLUSIONS}

Our magnetic measurements show that rare earth orthoferrites exhibit a non-zero spontaneous magnetic moment along the crystalline $b$ axis, $M_{b}$. These measurements are consistent with a picture where $M_{b}$ is contributed by both $\mathrm{Fe}^{3+}$ and $R^{3+}$ ions. The measurements suggest that the magnetic $R^{3+}$ ions suppress $M_{b}$ contribution of $\mathrm{Fe}^{3+}$ to zero above $200 \mathrm{~K}$. The polarization of $R^{3+}$ by the ordered $\mathrm{Fe}^{3+}$ ions alleviates this suppression of $M_{b}$, resulting in non-zero $M_{b}$ below $200 \mathrm{~K}$ that peaks at around $10^{-3} \mu_{\mathrm{B}} /$ f.u. in the spin reorientation region. Representation analysis of space groups shows that non-zero $M_{b}$ for $R \mathrm{FeO}_{3}$ systems implies an admixture of the $\Gamma_{3}$ magnetic phase to already well-documented $\Gamma_{2}$ or $\Gamma_{4}$. In the $\Gamma_{3}$ phase, both $\mathrm{Fe}^{3+}$ and $R^{3+}$ spins have a small ferromagnetic component along the crystalline $b$ axis, which is in the opposite direction for $\mathrm{Fe}^{3+}$ and $R^{3+}$ spins for the samples measured. The spin Hamiltonian of at least $\mathrm{YFeO}_{3}$ has to include the fourth or higher even order of spins to describe the $\Gamma_{3}$ phase. Our measurements may look at odds with numerous reports based on neutron techniques, reporting $M_{b}=0$. However, neutron techniques lack the sensitivity to measure the small non-zero $M_{b}$ reported here.

\section{ACKNOWLEDGMENTS}

Mohanad H. Mohammed was supported by the Higher Committee for Education Development (HCED) in Iraq through the provision of a Ph.D. scholarship. This work is partially supported by the National Natural Science Foundation of China (NNSFC, Nos. 11774217 and 51372149). Z. X. Cheng acknowledges the Australian Research Council for support (No. DP190100150). The authors gratefully acknowledge the beam time at the Australian Nuclear Science and Technology Organisation (ANSTO) through Grant Nos. P7362 and DB7158 and access to the ANSTO PPMS through Grant No. X6356.

\section{APPENDIX: CORRECTIONS}

Even though our crystals were of very high quality, a minute amount of twinning was observed. This, together with the sample misorientation in the magnetometer, results in non-zero projections of $M_{a}$ and $M_{c}$ onto the nominal $b$ axis of the sample (i.e., the magnetometer axis). Corrections of the values of $M_{b}$ for both of these artifacts are performed, as described below.

The observed twinning allows for minute projections of the magnetic moment along each of the crystalline axes onto both of the other axes. Therefore, the measured magnetic moments along the three nominal crystalline axes can be described as

$$
\begin{aligned}
& M^{\prime}{ }_{a}=M_{a}+\Gamma M_{b}+\Theta M_{c}+\Lambda M_{c}, \\
& M^{\prime}{ }_{c}=M_{c}+\Pi M_{a}+\chi M_{b}+\eta M_{a}, \\
& M^{\prime}{ }_{b}=M_{b}+\Xi M_{a}+\Sigma M_{c}+\Phi M_{c}+\Psi M_{a},
\end{aligned}
$$

where $M_{a}, M_{b}$, and $M_{c}$ are the true magnetic moments along the corresponding crystalline axes. The terms with $\Gamma, \Theta, \Pi, \chi, \Xi$, and $\Sigma$ are contributions to the measured moment by the twinned crystal volumes. They depend on the Euler angles and volume fraction of each twin. Their values are much smaller than 1 , as less than $1 \%$ of the crystal volume is occupied by the twins. The terms with $\Lambda, \eta$, 
$\Phi$, and $\Psi$ are contributions to the measured moment by the projections of $M_{a}$ and $M_{c}$ onto the axis of the magnetometer through the sample misalignment in the magnetometer. They represent cosines of the small misalignment angles. As an experiment shows that $M_{b} \ll M_{a}, M_{c}$, the contribution of $M_{b}$ to $M_{a}^{\prime}$ and $M_{c}^{\prime}$ due to the sample misalignment was neglected. Rearrangement of Eq. (A1) gives

$$
\begin{aligned}
& M_{a}^{\prime}=M_{a}+\Gamma M_{b}+(\Theta+\Lambda) M_{c}, \\
& M_{c}^{\prime}=M_{c}+\chi M_{b}+(\Pi+\eta) M_{a}, \\
& M_{b}^{\prime}=M_{b}+(\Xi+\Psi) M_{a}+(\Sigma+\Phi) M_{c} .
\end{aligned}
$$

Therefore, the combined contribution of the sample misalignment and twinning results in the measured moment along a particular axis to consist of a linear combination of the true moments along all the three crystalline axes. To obtain the true magnetic moment along the crystalline $b$ axis, we need to consider only $M_{b}^{\prime}$, which can be re-written as

$$
M_{b}^{\prime}=M_{b}+\alpha M_{a}+\beta M_{c} .
$$

Measured temperature dependence of $M_{a}$ and $M_{c}$ shows that $M_{a}=0$ at high temperatures and $M_{c}=0$ below the temperatures of spin reorientation (Fig. 4). If we assume that $M_{b}=0$, as the literature reports, $M_{b}^{\prime}(T)$ should be possible to fit with linear combinations of $M_{a}(T)$ and $M_{c}(T)$, as given by Eq. (A3). To achieve that, the initial value of $\alpha$ was obtained from an arbitrarily chosen lowtemperature value of $M_{a}$, where $M_{c}=0$, as $\alpha=M_{b}^{\prime} / M_{a}^{\prime}$. The initial value of $\beta$ was obtained at high temperatures, where $M_{a}=0$, as $\beta=M_{b}^{\prime} / M_{c}^{\prime}$. These values of $\alpha$ and $\beta$ were further refined through the fitting procedure to obtain the best agreement between the measured $M^{\prime}(T)$ and the fit by Eq. (A3) (assuming $M_{b}=0$ ). Obtaining a good fit to $M_{b}^{\prime}(T)$ at all temperatures would signify that $M_{b}=0$. However, if the fit cannot follow $M_{b}^{\prime}(T)$ at least at some temperatures, $M_{b}$ has to be non-zero at these temperatures. The temperature dependence of $M_{b}$ is then obtained as

$$
M_{b}(T)=M_{b}^{\prime}(T)-\alpha M_{a}(T)-\beta M_{c}(T) .
$$

The fitting coefficients $\alpha$ and $\beta$ depend on the crystal twinning and sample misalignment in the magnetometer, and they are not temperature-dependent. We assumed here that the twins have the same temperature dependence of $M_{a}, M_{b}$, and $M_{c}$ as the main crystal body. In this way, now in the temperature dependencies of magnetic moments, it was possible to eliminate the effects of twinning and sample misalignment to the measured $M_{b}$.

One has to stress that thus obtained $M_{b}(T)$ is not completely defined. It, in principle, depends on the choice of the temperature range for which the fit agrees with the measured $M_{b}^{\prime}(T)$, as $M_{a}^{\prime}$ and $M_{c}^{\prime}$ are not exactly zero at high and low temperatures, respectively. This means that the $M_{b}(T)$ obtained from Eq. (A4) can be slightly tilted, as defined approximately by a straight line connecting the points in the $M(T)$ diagram that gave us the initial estimates of $\alpha$ and $\beta$.

Equations (A1)-(A3) assume that the effects of crystal twinning are superimposed linearly to the measured magnetic moment.
However, spins at the interface between the twins and parent crystal can undergo complex re-arrangements. In the spin reorientation temperature range, spontaneous reorientation of the spins could result in complicated interactions at this interface. Such processes may contribute to the magnetic moment along the crystalline $b$ axis that is not proportional to $M_{a}$ or $M_{c}$, in which case Eqs. (A1)-(A3) would not be correct. However, we note that the largest values of $M_{b}$ were obtained outside the spin reorientation temperature range (Figs. 5 and 6). Therefore, this mechanism cannot be responsible for the obtained non-zero $M_{b}$, at least outside the spin reorientation range.

We now discuss the possibility that the twin boundary spins contribute to $M_{b}$ by themselves, without the need for spin reorientation. XRD data show that $90^{\circ}$ twins could produce non-zero $M_{b}$, whereby the $M_{a}$ of the twins is oriented along the $b$ axis of the parent crystal. The boundary between the twins and parent crystal would represent a form of Néel domain wall. Magnetic poles form at such a boundary, which could produce the measured $M_{b}$. These poles could have different temperature dependences than $M_{a}$ and $M_{c}$ and above equations would again not be satisfied. However, $M_{b}$ would then have to be non-zero at all temperatures, assuming these poles are the main contribution to $M_{b}$, as twins do not disappear from the crystal with a temperature change between 5 and $300 \mathrm{~K}$. This is contrary to the experimental findings (Fig. 6).

\section{REFERENCES}

${ }^{1}$ Y. Tokunaga, S. Iguchi, T. Arima, and Y. Tokura, Phys. Rev. Lett. 101, 097205 (2008).

${ }^{2}$ J. H. Lee, Y. K. Jeong, J. H. Park, M. A. Oak, H. M. Jang, J. Y. Son, and J. F. Scott, Phys. Rev. Lett. 107, 117201 (2011).

${ }^{3}$ E. Bousquet and A. Cano, J. Phys. Condens. Matter 28, 123001 (2016).

${ }^{4}$ E. Bousquet and N. Spaldin, Phys. Rev. Lett. 107, 197603 (2011).

${ }^{\mathbf{5}}$ H. J. Zhao, L. Bellaiche, X. M. Chen, and J. Íñiguez, Nat. Commun. 8, 14025 (2017).

${ }^{6}$ H. Katsura, N. Nagaosa, and A. V. Balatsky, Phys. Rev. Lett. 95, 057205 (2005).

${ }^{7}$ A. Kimel, B. Ivanov, R. Pisarev, P. Usachev, A. Kirilyuk, and T. Rasing, Nat. Phys. 5, 727 (2009).

${ }^{8}$ S. Wienholdt, D. Hinzke, and U. Nowak, Phys. Rev. Lett. 108, 247207 (2012).

${ }^{9}$ M. R. Freeman and Z. Diao, Nat. Photonics 6, 643 (2012).

${ }^{10} \mathrm{~S}$. Mangin, D. Ravelosona, J. Katine, M. Carey, B. Terris, and E. E. Fullerton, Nat. Mater. 5, 210 (2006)

${ }^{11}$ T. Seifert et al., Nat. Photonics 10, 483 (2016).

${ }^{12}$ R. L. White, J. Appl. Phys. 40, 1061 (1969).

${ }^{13}$ M. Eibschütz, S. Shtrikman, and D. Treves, Phys. Rev. 156, 562 (1967).

${ }^{14}$ T. Yamaguchi, J. Phys. Chem. Solids 35, 479 (1974).

${ }^{15}$ D. Treves, Phys. Rev. 125, 1843 (1962).

${ }^{16}$ E. Wollan and W. Koehler, Phys. Rev. 100, 545 (1955).

${ }^{17}$ E. Bertaut, J. Appl. Phys. 33, 1138 (1962).

${ }^{18}$ I. Dzyaloshinsky, J. Phys. Chem. Solids 4, 241 (1958).

${ }^{19}$ T. Moriya, Phys. Rev. Lett. 4, 228 (1960).

${ }^{20}$ L. Bellaiche, Z. Gui, and I. A. Kornev, J. Phys. Condens. Matter 24, 312201 (2012).

${ }^{21}$ K. P. Belov, A. K. Zvezdin, A. M. Kadomtseva, and R. Levitin, Sov. Phys. Usp. 19, 574 (1976).

${ }^{22}$ D. Treves, J. Appl. Phys. 36, 1033 (1965).

${ }^{23}$ L. Tsymbal, V. Kamenev, Y. B. Bazaliy, D. Khara, and P. Wigen, Phys. Rev. B 72, 052413 (2005).

${ }^{\mathbf{2 4}}$ L. Tsymbal, Y. B. Bazalǐ̌, G. Kakazeĭ, Y. I. Nepochatykh, and P. Wigen, Low Temp. Phys. 31, 277 (2005). 
${ }^{25}$ L. T. Tsymbal, Y. B. Bazaliy, V. N. Derkachenko, V. I. Kamenev, G. N. Kakazei, F. J. Palomares, and P. E. Wigen, J. Appl. Phys. 101, 123919 (2007).

${ }^{26}$ E. Gyoroy, J. Remeika, and F. Hagedorn, J. Appl. Phys. 39, 1369 (1968).

${ }^{27}$ D. Georgiev, K. Krezhov, and V. Nietz, Solid State Commun. 96, 535 (1995).

${ }^{28}$ J. Bartolomé, E. Palacios, M. Kuz'min, F. Bartolomé, I. Sosnowska, R. Przeniosło, R. Sonntag, and M. Lukina, Phys. Rev. B 55, 11432 (1997).

${ }^{29}$ W. Sławiński, R. Przeniosło, I. Sosnowska, and E. Suard, J. Phys. Condens. Matter 17, 4605 (2005).

${ }^{30} \mathrm{R}$. Hornreich and I. Yaeger, Int. J. Magn. 4, 6 (1973).

${ }^{31}$ X.-P. Yuan, Y.-K. Tang, Y. Sun, and M.-X. Xu, J. Appl. Phys. 111, 053911 (2012).

${ }^{32}$ Y. Tokunaga, N. Furukawa, H. Sakai, Y. Taguchi, T.-H. Arima, and Y. Tokura, Nat. Mater. 8, 558 (2009).

${ }^{33}$ T. Chatterji, A. Stunault, and P. Brown, J. Phys. Condens. Matter 29, 385802 (2017).

${ }^{34}$ H. Pinto and H. Shaked, Solid State Commun. 10, 663 (1972).

${ }^{35}$ E. Bertaut, Acta Crystallogr. A 24, 217 (1968).

${ }^{36} \mathrm{H}$. Shen, J. Xu, M. Jin, and G. Jiang, Ceram. Int. 38, 1473 (2012).

${ }^{37}$ M. Shang, C. Zhang, T. Zhang, L. Yuan, L. Ge, H. Yuan, and S. Feng, Appl. Phys. Lett. 102, 062903 (2013).

${ }^{38}$ I. Jacobs, H. F. Burne, and L. M. Levinson, J. Appl. Phys. 42, 1631 (1971).

${ }^{39}$ G. Song, J. Jiang, B. Kang, J. Zhang, Z. Cheng, G. Ma, and S. Cao, Solid State Commun. 211, 47 (2015).
${ }^{40}$ S. Yuan, Y. Wang, M. Shao, F. Chang, B. Kang, Y. Isikawa, and S. Cao, J. Appl. Phys. 109, 07E141 (2011).

${ }^{41}$ W. Koehler, E. Wollan, and M. Wilkinson, Phys. Rev. 118, 58 (1960).

${ }^{42}$ H. Shen, Z. Cheng, F. Hong, J. Xu, S. Yuan, S. Cao, and X. Wang, Appl. Phys. Lett. 103, 192404 (2013).

${ }^{43}$ R. Przeniosło, I. Sosnowska, M. Loewenhaupt, and A. Taylor, J. Magn. Magn. Mater. 140, 2151 (1995).

${ }^{44}$ S. Yuan, W. Ren, F. Hong, Y. Wang, J. Zhang, L. Bellaiche, S. Cao, and G. Cao, Phys. Rev. B 87, 184405 (2013).

${ }^{45}$ H. Shen, J. Xu, A. Wu, J. Zhao, and M. Shi, Mater. Sci. Eng. B 157, 77 (2009).

${ }^{46}$ Y. B. Bazaliy, L. Tsymbal, G. Kakazei, A. Izotov, and P. Wigen, Phys. Rev. B 69, 104429 (2004).

${ }^{47}$ G. Deng, P. Guo, W. Ren, S. Cao, H. E. Maynard-Casely, M. Avdeev, and G. J. McIntyre, J. Appl. Phys. 117, 164105 (2015).

${ }^{48}$ A. Balbashov, G. Kozlov, S. Lebedev, A. Mukhin, A. Y. Pronin, and A. Prokhorov, JETP Lett. 95, 1092 (1989).

${ }^{49} \mathrm{G}$. Vorob'ev, A. Kadomtseva, I. Krynetkii, and A. Mukhin, Sov. Phys. JETP 95, 1049 (1989).

${ }^{50}$ Z. Zhao, X. Zhao, H. Zhou, F. Zhang, Q. Li, C. Fan, X. Sun, and X. Li, Phys. Rev. B 89, 224405 (2014).

${ }^{51}$ S. Bujko, D. Georgiev, K. Krezhov, V. Nietz, and G. Passage, J. Phys. Condens. Matter 7, 8099 (1995). 